\title{
Evaluation of Parallel-Element, Variable-Impedance, Broadband Acoustic Liner Concepts
}

\author{
M. G. Jones* and B. M. Howerton ${ }^{\dagger}$ \\ NASA Langley Research Center, Hampton, VA 23681 \\ E. Ayle \\ Hexcel Corporation, Casa Grande, AZ 85130
}

\begin{abstract}
Recent trends in aircraft engine design have highlighted the need for acoustic liners that provide broadband sound absorption with reduced liner thickness. Three such liner concepts are evaluated using the NASA normal incidence tube. Two concepts employ additive manufacturing techniques to fabricate liners with variable chamber depths. The first relies on scrubbing losses within narrow chambers to provide acoustic resistance necessary for sound absorption. The second employs wide chambers that provide minimal resistance, and relies on a perforated sheet to provide acoustic resistance. The variable-depth chambers used in both concepts result in reactance spectra near zero. The third liner concept employs mesh-caps (resistive sheets) embedded at variable depths within adjacent honeycomb chambers to achieve a desired impedance spectrum. Each of these liner concepts is suitable for use as a broadband sound absorber design, and a transmission line model is presented that provides good comparison with their respective acoustic impedance spectra. This model can therefore be used to design acoustic liners to accurately achieve selected impedance spectra. Finally, the effects of increasing the perforated facesheet thickness are demonstrated, and the validity of prediction models based on lumped element and wave propagation approaches is investigated. The lumped element model compares favorably with measured results for liners with thin facesheets, but the wave propagation model provides good comparisons for a wide range of facesheet thicknesses.
\end{abstract}

\section{Introduction}

A significant desire for reduced aircraft noise in the vicinity of airports has led the International Civil Aviation Organization (ICAO) and Federal Aviation Administration (FAA) to impose stringent aircraft noise requirements. In response to these requirements, NASA's Fundamental Aeronautics Program has set a goal to develop noise reduction concepts sufficient to confine objectionable aircraft noise within the airport boundaries. Clearly, this will require significant advances in innovative aircraft noise reduction technology.

Aircraft noise can conveniently be divided into two components, propulsion noise and airframe noise. Of these, propulsion noise is dominant for the takeoff condition, and is similar in magnitude with airframe noise for the approach condition. ${ }^{1}$ The two key contributors to propulsion noise are jet noise and fan noise. Significant progress has been made in the reduction of jet noise via the use of high bypass-ratio engines, while modifications to the fan geometry (e.g., lean and sweep of the fan blades or rotor-stator spacing $)^{2,3}$ have been combined with conventional, perforate-over-honeycomb acoustic liners installed in the interior walls of aircraft engine nacelles to significantly reduce the tonal component of fan noise. This has caused the dominant spectral character of fan noise to transition from tonal to broadband. As a result, broadband fan noise remains a dominant source of objectionable noise for vicinities near airports, and significant further

* Senior Research Scientist, Research Directorate, Structural Acoustics Branch, AIAA Associate Fellow.

$\dagger$ Research Scientist, Research Directorate, Structural Acoustics Branch.

${ }^{\ddagger}$ Senior Design Engineer, Research and Technology Division. 
advances will be required if the goal to confine aircraft objectionable noise within airport boundaries is to be achieved.

One key element for broadband fan noise reduction is the usage of multi-layer acoustic liners mounted in the walls of the aircraft engine nacelle. When designed properly, the addition of extra layers (one layer is generally considered to be the combination of an air gap and a resistive element, e.g., a perforated sheet) increases the absorptive bandwidth of the liner. However, due to constraints on the thickness of the nacelle wall, acoustic liners generally contain no more than two layers. ${ }^{4}$ These two-layer liners provide sound absorption over a wider frequency range than can be achieved with conventional single-layer liners. However, further advances are needed to increase the amount of asborption over a wider frequency range. The development of acoustic liner configurations that improve broadband noise reduction with reduced liner thickness is critical in this endeavor.

The purpose of the current study is to investigate three such broadband acoustic liner concepts, each of which has been made available via recent advances in manufacturing techniques. The first liner concept uses additive manufacturing technology to create parallel-element, variable-depth, open chambers to achieve variable impedance over the liner surface. ${ }^{5,6}$ These chambers are sufficiently narrow to provide viscous scrubbing losses along their interior walls, such that they do not require the inclusion of a facesheet. The second liner concept is similar to the first, but with much wider chambers. As a result, these liners require a facesheet to provide the necessary acoustic resistance and to avoid self-noise due to interactions between the open chambers and the flow environment just above the liner surface. Each of these two concepts incorporates variable-depth chambers to achieve broadband sound absorption. The third liner concept uses embedded mesh-caps bonded into the honeycomb cells ${ }^{7}$ as the septum of a two-layer, perforate-over-honeycomb liner. A variable impedance is achieved over the liner surface by varying the insertion depth and flow resistance of mesh-caps embedded in adjacent honeycomb cells.

Each of these three liner concepts is evaluated in a no-flow environment via tests conducted with the NASA normal incidence tube (NIT). Models are used to predict the impedance spectra for a number of test liners, and these spectra are compared with those measured with the NIT. Comparisons of measured and predicted impedance spectra are used to assess the efficacy of two impedance prediction models. A good comparison between measured and predicted impedance spectra provides confidence in using the models to design acoustic liners optimized for a selected application. Section II provides a description of the experimental method used to evaluate the liner configurations, and also provides a description of each of the test liners used in this study. The impedance prediction models are provided in Section III. Comparisons of predicted and measured impedance spectra for these liners are provided in Section IV, along with a discussion of the key features. Finally, concluding remarks are presented in Section V.

\section{Experimental Method}

\section{A. Normal Incidence Tube Measurements}

Each liner used in this investigation is tested in the NASA Langley normal incidence tube (NIT), a 2-in $\times 2$ in waveguide (see sketch in Fig. 1). It contains six 120-W compression drivers to generate a plane-wave sound field that impinges on the surface of the liner and combines with reflections from the liner to create a standing wave pattern. The Two-Microphone Method $^{8,9}$ is used to measure the complex acoustic pressures at two prescribed distances from the liner surface, such that the frequency dependence of the no-flow acoustic impedance of the liner can be computed. Tonal tests (one frequency at a time) are conducted for source frequencies of 0.4 to $3.0 \mathrm{kHz}$ in $0.2 \mathrm{kHz}$ increments, with source sound pressure levels (SPLs) of 120 and $140 \mathrm{~dB}$ at the reference microphone. These tones are generated by a computer-controlled function generator. Two amplitudes are used so that test liner nonlinearities can be evaluated by determining whether the impedance is a function of the reference SPL.

\section{B. Test Liners}

A general rule of thumb for local-reacting, variable-impedance liners is that the impedance variability can be ignored (i.e., impedance can be assumed uniform) if the geometry variability is confined within a small 


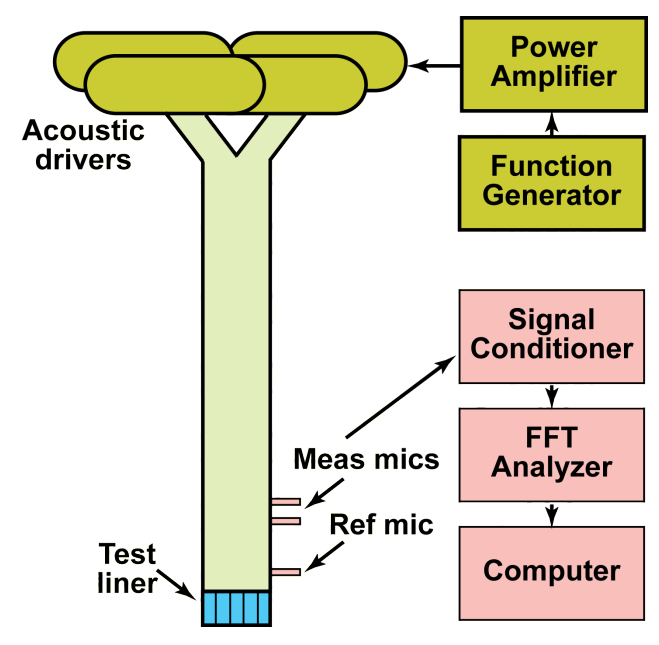

Figure 1: Sketch of NASA normal incidence tube (NIT).

portion of the full liner, and then repeated over the full extent of the liner. As long as each unit cell is less than one-eighth (often relaxed to one quarter) of a wavelength of the source frequency, then a uniform impedance spectrum can be achieved. Each of the liner concepts included in this study was selected because they can be designed to achieve significant impedance variability over a small spatial extent. Also, the broadband liners used in this study were designed for optimum sound absorption within the frequency range of 0.4 to $3.0 \mathrm{kHz}$, such that they could be properly evaluated using the NIT.

Three types of acoustic liners are used in this study. The first consists of multiple parallel, variable-depth, narrow chambers. The chamber diameter is on the same order of magnitude as the diameter of perforates in conventional liner facesheets. It is therefore assumed to be sufficiently small such that this type of liner does not require a facesheet. One example of a narrow-chamber liner configuration that has been heavily investigated by $\mathrm{NASA}^{10}$ is the ceramic tubular, uniform-depth, narrow-chamber liner shown in Fig. 2. The second liner type is very similar, except the chambers are significantly wider, such that a facesheet would be required to inhibit mean flow from entering and exiting the chambers. The last liner type is a multi-layer liner that incorporates mesh-caps embedded into honeycomb core. In its most basic implementation, the insertion depths of the mesh-caps are uniform for the entire liner, as shown in Fig. 3. Consistent results were achieved with a number of liners of each type. Thus, for the sake of brevity, results are provided for a limited number of these liners.

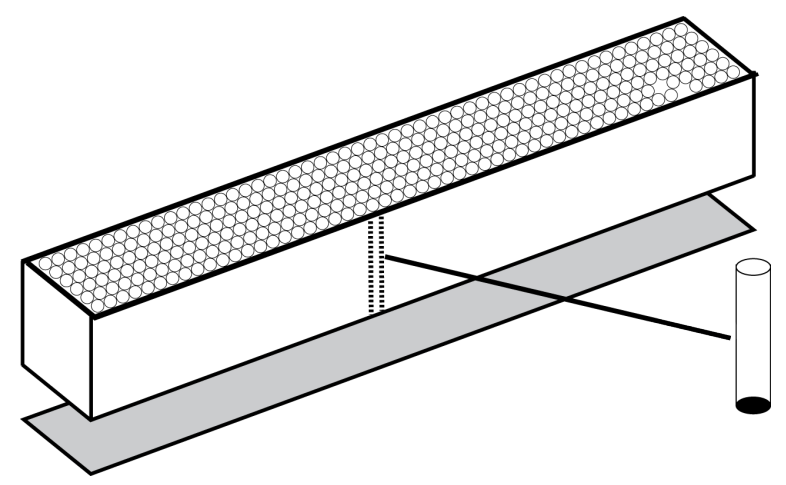

(a) Ceramic tubular liner.

Figure 2: Sketch of ceramic tubular liner. 


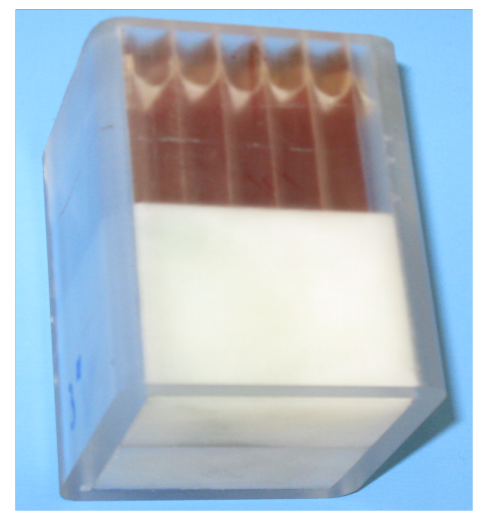

(a) Side view.

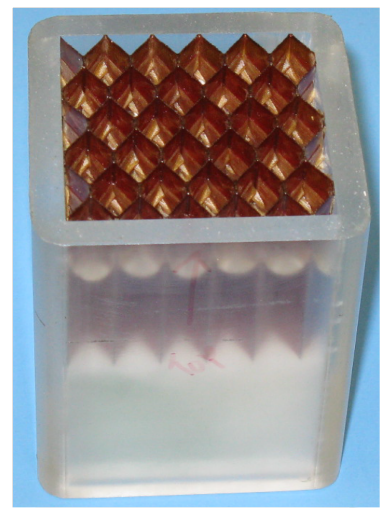

(b) Top view.

Figure 3: Photographs of sample with single mesh-cap insert; facesheet not shown.

\section{Parallel-Element, Variable-Depth Liners with Narrow Chambers}

In 1995, Parrott and Jones ${ }^{6}$ presented results for a group of parallel-element, narrow-chamber liners constructed from ceramic tubular material with a surface porosity of $57 \%$. They demonstrated the impedance of liners constructed in this manner could be predicted with great confidence, and could be designed with variable-depth chambers to achieve a nearly uniform impedance spectrum over a significant frequency range. For these reasons, NASA continues to use ceramic tubular liners to validate impedance eduction processes. ${ }^{10}$ However, this material is very heavy and brittle, and is not practical for use in aircraft engine nacelle liners.

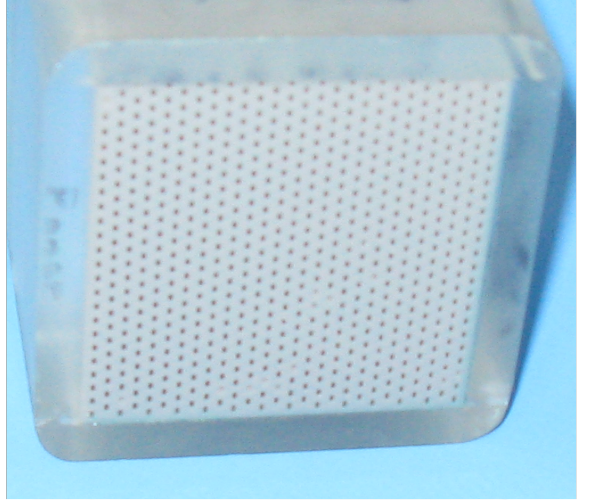

(a) Photo of narrow-chamber liner surface.

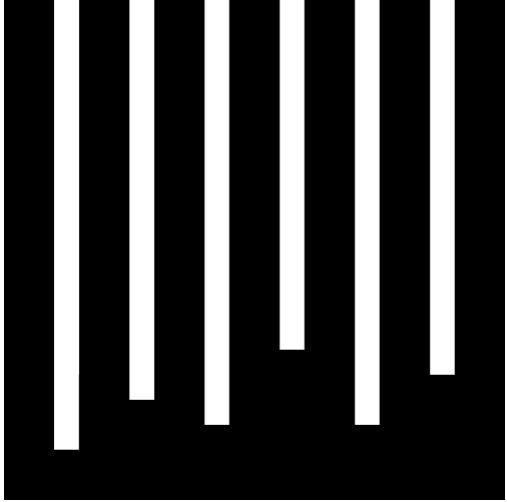

(b) Sketch of narrow-chamber liner with variable depth chambers.

Figure 4: Narrow-chamber liner.

This liner design concept (see Fig. 4) is included in the current study to determine whether modern materials and manufacturing processes can be used to achieve results of similar quality to those achieved with ceramic tubular material. Stereolithography allows the forming of solid, 3-D models from liquid plastic resin by application of laser light to harden the resin. A computer-controlled laser beam is scanned over the surface of the resin vat, tracing the area of one layer of the part to be formed and causing that layer to harden. Successive layers are built up until the entire part is formed. Part tolerances are to within \pm 0.0025 in of the target dimensions. The samples fabricated for this test were formed using a resin that affords sufficient strength (tensile modulus of about 350,000 psi) to maintain acoustic isolation between neighboring channels. 
These narrow-chamber liners derive their acoustic resistance from viscous losses within the narrow chambers. They remain too heavy for extensive use in aircraft applications, but are ideal for exploratory laboratory studies. As mentioned earlier, a number of constant-depth and variable-depth configurations were evaluated. The quality of the results achieved with each of these liners was very good. The two configurations listed in Table 1, one with constant-depth chambers, and the other with variable-depth chambers, are used to provide representative results. The constant-depth liner contains 177 chambers, each with a diameter of 0.07 inches and a depth of 1.69 inches. The variable-depth liner contains chambers with nine unique depths, all with diameters of 0.07 inches. This group of nine chambers is repeated thirty times within the 2-inch $\times 2$-inch liner, resulting in a surface porosity of $26 \%$. As an aside, it should be noted that these samples were initially fabricated for a different study, which is the reason the two samples contain a different number of chambers.

\begin{tabular}{|c|c|c|}
\hline Liner \# & NC1 & NC2 \\
\hline Diameter (inches) & 0.07 & 0.07 \\
\hline Chamber Depths (inches) & 1.69 & $1.12,1.24,1.36,1.50,1.65,1.81,1.99,2.19,2.41$ \\
\hline Porosity (\%) & 17 & 26 \\
\hline Total \# Chambers & 168 & 247 \\
\hline
\end{tabular}

Table 1: Narrow-chamber test liners.

\section{Parallel-Element, Variable-Depth Liners with Wide Chambers}

There is one key difference between parallel-element, variable-depth liners consisting of narrow and wide chambers. Whereas the acoustic resistance of narrow-chamber liners is derived from viscous losses within the narrow chambers, the chamber diameter in wide-chamber liners is assumed to be sufficiently large to virtually eliminate these viscous losses. Instead, wide-chamber liners depend on a facesheet (e.g., perforated sheet) to provide the acoustic resistance. These liners require fewer chambers than their narrow-chamber counterparts, and are therefore expected to be lighter and simpler to fabricate. However, due to the increased chamber diameter, variable-depth chambers cannot be grouped as tightly, which is expected to limit controllability of the impedance spectrum at the liner surface (i.e., more difficult to confine the impedance variability within a small portion of a wavelength of the source frequency).

\begin{tabular}{|c|c|cc|}
\hline Liner \# & WC1 & \multicolumn{2}{|c|}{ WC2 } \\
\hline Chamber Depths (inches) & 1.50 & $(1.14,1.36,2.35)$ & $(1.64,1.96,2.82,3.39)$ \\
\hline \# Chambers at Given Depth(s) & 25 & 3 of each & 4 of each \\
\hline
\end{tabular}

Table 2: Cores for wide-chamber test liners.

\begin{tabular}{|c|c|c|c|c|}
\hline Facesheet \# & F1 & F2 & F3 & F4 \\
\hline Hole Diameter (inches) & 0.035 & 0.035 & 0.035 & 0.035 \\
\hline Sheet Thickness (inches) & 0.025 & 0.500 & 0.100 & 0.200 \\
\hline Porosity (\%) & 10 & 10 & 10 & 10 \\
\hline
\end{tabular}

Table 3: Facesheets for wide-chamber test liners.

Each 2 -inch $\times 2$-inch liner contains twenty-five 0.375 -inch $\times 0.375$-inch chambers, and may be combined with interchangeable facesheets to create multiple liner configurations (see Fig. 5). Two cores (see Tab. 2) are used to demonstrate key features of this liner concept. The first contains twenty-five constant-depth chambers, similar to configurations currently used in aircraft engine nacelle liners. The second contains two 
groups of chambers with variable depths, with three and four chambers per group, respectively. Three sets of the first group and four sets of the second group are combined for a total of twenty-five chambers. Four facesheets (see Tab. 3) with identical hole diameters and porosities are chosen to cover a range of sheet thicknesses from 0.025 inches to 0.200 inches. Additional facesheets were also examined, to cover ranges of hole diameters and porosities, but the sheet thickness variability is chosen for presentation because it provided the most interesting features. However, it should be noted that variability of any of these parameters can be used to achieve a variable-impedance liner configuration.

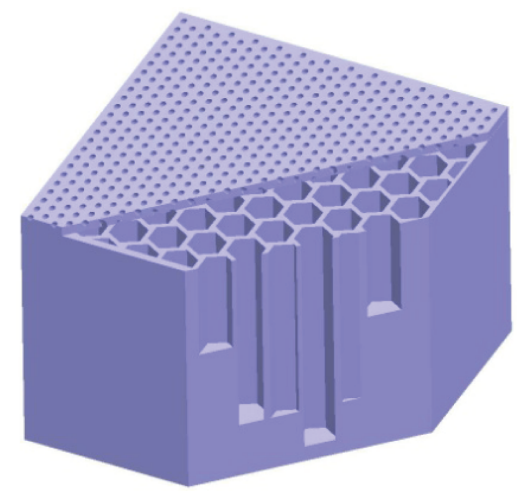

Figure 5: Cutaway view of variable-depth, wide-chamber liner with perforated facesheet.

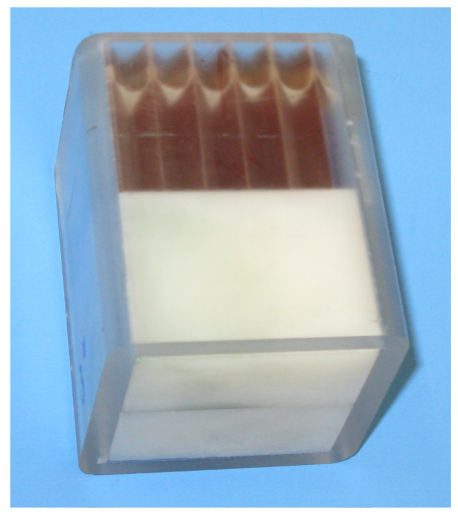

(a) Two-layer mesh-cap liner.

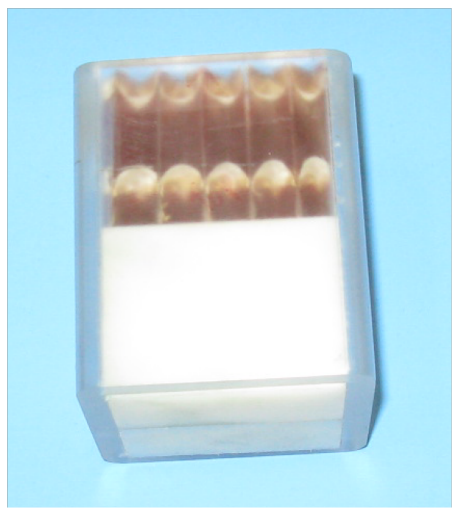

(b) Three-layer mesh-cap liner.

Figure 6: Photos of mesh-cap liners.

\section{Multi-Layer Liner with Mesh-Caps}

The last type of liner used in this study incorporates the Hexcel Acousti-Cap ${ }^{\circledR}$ product, which has been designed to support "Individual Cell Treatments." This concept allows the acoustic liner to be customized such that the surface impedance of each individual cell is independently controlled. This is achieved by the combination of parameters used to set the impedance in each cell. Available options include:

1. Each honeycomb cell can contain zero, one (Fig. 6a) or two (Fig. 6b) buried septa.

2. The depth of each septum can vary from cell to cell. Thus, either uniform-depth or variable-depth configurations can be constructed.

3. The DC flow resistance of each septum can vary from cell to cell. This allows the acoustic resistance of each cell to be independently controlled. 
4. The combination of one or both variables can be customized into a grid pattern of different septum depths or resistances within the acoustic panel.

Punch and die tooling are used to cut and form the Acousti-Cap ${ }^{\circledR}$ shape from a continuous web of PEEK mesh (see Fig. 7). The mesh material is shaped for insertion into the hexagonal shape, and a punch is used to form the mesh in the forming die and then to insert the formed mesh-cap into the honeycomb core. The Acousti-Cap ${ }^{\circledR}$ septum is anchored to the honeycomb cell wall with an adhesive bond at the chosen depth. The product is formable because the septum is a flexible mesh, yet the honeycomb maintains its full strength because it does not have to be spliced at each septum. The test liners used in this study are fabricated with 0.375-inch diameter honeycomb core material. Acoustic mesh material with DC flow resistances from 300 to 1200 MKS Rayls were considered. For convenience, these buried septa are referenced as "mesh-caps" in the remainder of this paper. As shown in Figs. 6 and 8, they can be inserted at uniform or variable depths.

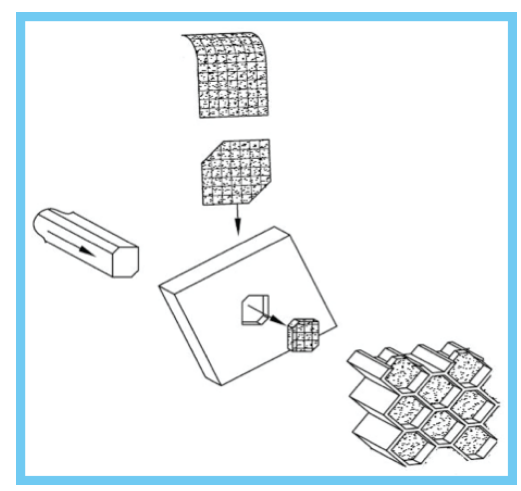

Figure 7: Sketch of punch and die process to cut and form mesh into proper dimensions for honeycomb cell.

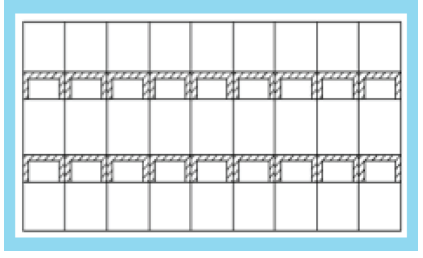

(a) Uniform-depth septa for threelayer liner.

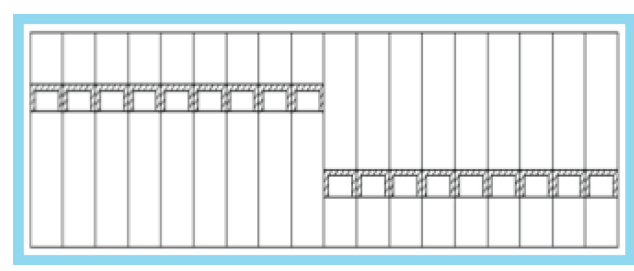

(b) Variable-depth septa for two-segment liner.

Figure 8: Sketches of two configurations with buried septa.

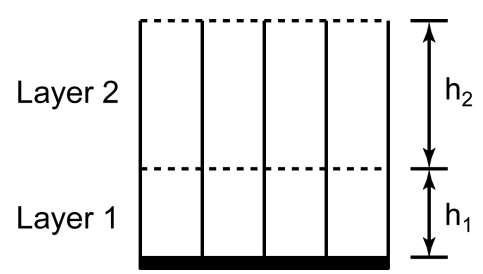

(a) Two-layer configuration.

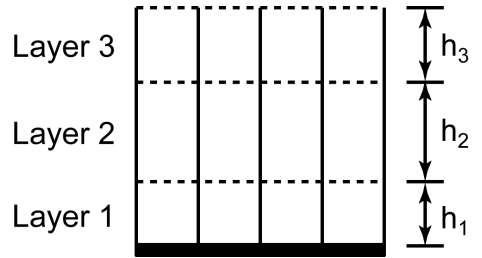

(b) Three-layer configuration.

Figure 9: Sketch of multi-layer configurations.

Multiple two- and three-layer liners (see Fig. 9) were tested in the NASA normal incidence tube (NIT), with very consistent results. For the sake of brevity, results presented in this report are confined to one two-layer liner and one three-layer liner that are representative of all of the liners tested. The components 
of these two liners are provided in Tab. 4. Note that the table does not contain entries for the facesheets of these liners. The liners used in this portion of the study were fabricated without backplates or facesheets. Each liner was mounted into a 2 -in $\times 2$-in sleeve with the same length as the multi-layer liner. This assembly was mounted onto a 1-in thick, brass backplate for the NIT tests. Additional tests were conducted with a perforated facesheet, but the current results are confined to those configurations without a facesheet.

\begin{tabular}{|c|c|c|}
\hline Liner $\#$ & MC1 & MC2 \\
\hline Layer 3 height, $h_{3}$, inches & - & 0.3 \\
\hline Layer 2 sheet DC flow resistance, MKS Rayls & - & 300 \\
\hline Layer 2 height, $h_{2}$, inches & 0.5 & 0.7 \\
\hline Layer 1 sheet DC flow resistance, MKS Rayls & 700 & 300 \\
\hline Layer 1 height, $h_{1}$, inches & 1.0 & 0.5 \\
\hline
\end{tabular}

Table 4: Components of multi-layer, mesh-cap liners.

\section{Impedance Prediction Models}

The impedance prediction models used in this study are based on the transmission line model discussed in detail in an earlier paper. ${ }^{6}$ The normalized surface impedance spectra presented by each individual chamber of the liner are computed separately. These impedance spectra are then combined to determine an effective surface impedance spectrum that is assumed uniform across the liner surface.

\section{A. Single Chamber Computations}

The transmission line model for predicting the surface acoustic impedance of an individual liner chamber is briefly described herein with the aid of Fig. 10. This figure provides a sketch of a single chamber of a liner with two air gaps (sections with heights of $h_{1}$ and $h_{3}$ ) and two perforate sheets (thicknesses of $h_{2}$ and $h_{4}$ ). The thickness of each perforate sheet is exaggerated to demonstrate distinct features of the models used in this study. This model treats each unique portion of the liner chamber separately. In the ensuing discussion, these unique portions (confined to air gaps and perforated sheets in this example) are treated as individual "computational layers."

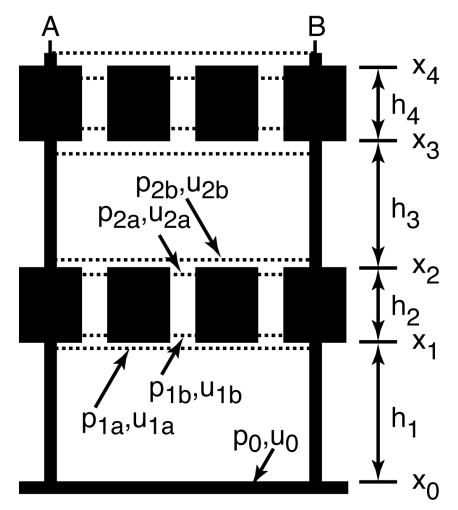

Figure 10: Sketch of single chamber of a liner with key parameters for transmision line impedance prediction model.

For each of the three liner concepts considered in this study, the transmission line computation is initiated by assuming the backplate at $x_{0}$ to be rigid and impervious. The acoustic pressure and particle velocity are 
given as

$$
\left(\begin{array}{l}
p_{0} \\
u_{0}
\end{array}\right)=\left(\begin{array}{l}
1 \\
0
\end{array}\right)
$$

which yields an infinite impedance at this location. All acoustic pressures and particle velocities are normalized by $\rho c^{2}$ and $c$, respectively, where the density of the air and the speed of sound are for the prevailing static pressure and air temperature. Changes in the acoustic pressure and particle velocity across a computational layer are computed via

$$
\left(\begin{array}{l}
p_{n+1} \\
u_{n+1}
\end{array}\right)=\left(\begin{array}{ll}
T_{11} & T_{12} \\
T_{21} & T_{22}
\end{array}\right)\left(\begin{array}{l}
p_{n} \\
u_{n}
\end{array}\right)
$$

where the transmission coefficients $\left(T_{11}, T_{12}, T_{21}\right.$ and $\left.T_{22}\right)$ depend on the modeling approach and the type of layer being considered.

For open layers (air gaps), the transmission coefficients are given by

$$
T_{11}=T_{22}=\cosh (k \Gamma h) ; T_{12}=\zeta_{c} \sinh (k \Gamma h) ; T_{21}=\zeta_{c}^{-1} \sinh (k \Gamma h)
$$

where $k=\omega / c$ is the free-space wavenumber, $\omega$ is the angular frequency, and $h$ is the layer thickness. Also, impedances are normalized by the characteristic impedance of air, $\rho c$. The propagation constant, $\Gamma$, and characteristic impedance, $\zeta_{c}$, are computed as

$$
\Gamma=\sqrt{\frac{J_{0}\left(i^{3 / 2} s\right)}{J_{2}\left(i^{3 / 2} s\right)}} \sqrt{\frac{\gamma}{n_{\Gamma}}} ; \quad \zeta_{c}=\frac{-i}{\Gamma} \frac{J_{0}\left(i^{3 / 2} s\right)}{J_{2}\left(i^{3 / 2} s\right)}
$$

where

$$
n_{\Gamma}=\left[1+\frac{\gamma-1}{\gamma} \frac{J_{0}\left(i^{3 / 2} \sigma s\right)}{J_{0}\left(i^{3 / 2} \sigma s\right)}\right]^{-1}
$$

$i=\sqrt{-1}$ is the imaginary unit, $s=\left(d_{c} / 2\right) \sqrt{\rho_{s} \omega / \mu}$ is the shear wave number, and $d_{c}, \rho_{s}, \omega, \mu, \sigma^{2}$ and $\gamma$ are the channel diameter, static density, angular frequency, coefficient of viscosity, Prandtl number and specific heat ratio, respectively. Note that if a liner chamber only contains a single open computational layer (air gap), the normalized surface impedance for that chamber is computed as

$$
\zeta_{c h}=\frac{p_{1}}{u_{1}}
$$

For those cases where the liner chamber contains more than one computational layer, the acoustic pressure and particle velocity computed at the top of the first computational layer $\left(p_{1}\right.$ and $\left.u_{1}\right)$ are input back into Eq. 2 to compute the impedance change across the next computational layer.

Two approaches are considered for computing the change in impedance across perforated sheets. The first assumes the perforated sheet to be a purely resistive lumped element that is too thin to support wave propagation. For this approach, the transmission coefficients for Eq. 2 are given by

$$
T_{11}=T_{22}=1 ; T_{12}=\frac{R_{f}}{\rho c} ; T_{21}=0
$$

where the normalized DC flow resistance, $R_{f} / \rho c$, across the perforated sheet is often estimated from a semiempirical model (e.g., the Two-Parameter Impedance Prediction Model ${ }^{11}$ ). In the results provided below, impedances predicted using this approach are designated as "LE" (lumped element).

The second approach assumes the perforated sheet to be sufficiently thick to support wave propagation. Figure 10 is particularly useful to describe this approach. It is assumed that Eqs. 2 and 3 have been used to determine the acoustic pressure and particle velocity $\left(p_{1 a}\right.$ and $\left.u_{1 a}\right)$ at the top of the lower air gap. The acoustic pressure and acoustic mass flow are assumed to be constant across the $\{1 a, 1 b\}$ interface, such that

$$
p_{1 b}=p_{1 a} ; \quad N S_{1 b} u_{1 b}=S_{1 a} u_{1 a}
$$


where $N$ is the number of orifices ( 3 in this example) connected to a single air cavity, $S_{1 a}$ is the cross-sectional area at the top of the air cavity (denoted by the long dashed line at the top of the cavity) and $S_{1 b}$ is the cross-sectional area within a single perforate orifice. Next, the wave propagation within the single orifice is computed using Eqs. 2 and 3, where the orifice diameter is used as the "channel diameter." Again, the acoustic pressure and acoustic mass flow are assumed to be constant across the $\{2 a, 2 b\}$ interface, such that

$$
p_{2 b}=p_{2 a} ; \quad S_{2 b} u_{2 b}=N S_{2 a} u_{2 a}
$$

Results achieved using this model for perforate sheets are designated as "WP" (wave propagation) in the results provided below.

Regardless of which approach is used to model perforated sheets (for those configurations that contain them), the process described above is repeated until the acoustic pressure and particle velocity are determined at the surface of the chamber (denoted by the dashed line with endpoints $A$ and $B$ ). The normalized surface impedance for the chamber is computed as

$$
\zeta_{c h}=\frac{p_{n}}{u_{n}}
$$

where $n$ represents the number of computational layers in the chamber.

\section{B. Liner Surface Impedance Computation}

The surface impedance spectra of the individual chambers are combined to compute the effective impedance across the liner surface. For this computation, it is preferable to use acoustic admittance $\left(\beta_{c h}=1 / \zeta_{c h}\right)$. The effective admittance across a selected extent of liner surface is given by

$$
\beta_{s}=\Omega \sum_{i=1}^{N_{c h}} \beta_{c h}
$$

where $N_{c h}$ represents the number of chambers that combine to form the liner and $\Omega$ is the surface open area ratio (porosity) of the liner. The uniform, effective, surface impedance of the liner is then given by $\zeta_{s}=1 / \beta_{s}$.

\section{Results and Discussion}

The purpose of the current study is to evaluate three broadband acoustic liner concepts in a no-flow environment via tests conducted with the NASA normal incidence tube (NIT). Impedance models are used to predict the impedance spectra for each liner, and these spectra are compared with those measured with the NIT using the Two-Microphone Method. Comparisons of measured and predicted impedance spectra are used to assess the efficacy of two impedance prediction models. In all of the successive figures, the measured data are provided in $0.2 \mathrm{kHz}$ increments from 0.4 to $3.0 \mathrm{kHz}$, and the predicted results are provided in $0.025 \mathrm{kHz}$ increments over the same frequency range.

\section{A. Parallel-element, variable-depth, narrow-chamber liners}

Results are provided for two narrow-chamber liners, each consisting of round chambers with 0.07-inch diameters. The impedance $(\zeta=\theta+i \chi)$ spectra for these liners are predicted using Eqs. 1-6. Figure 11 provides a comparison of predicted and measured impedance spectra for the constant-depth liner (NC1). For all but the lowest frequencies, the comparison is excellent. The normalized resistance, $\theta$, is slightly less than 0.5 for frequencies near resonance (frequency at which positive-going zero crossing occurs in the reactance spectrum), and increases at the low and high frequency extremes near the anti-resonance frequencies. As expected for a constant-depth liner, the normalized reactance, $\chi$, is well approximated by $-\cot (k h)$. 


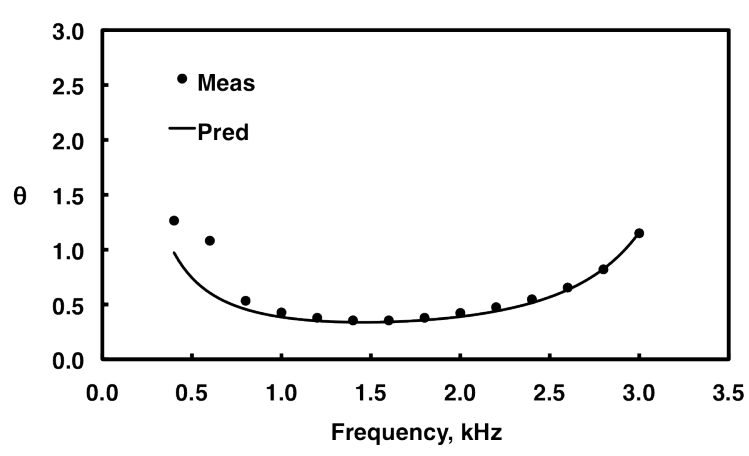

(a) Normalized resistance, $\theta$.

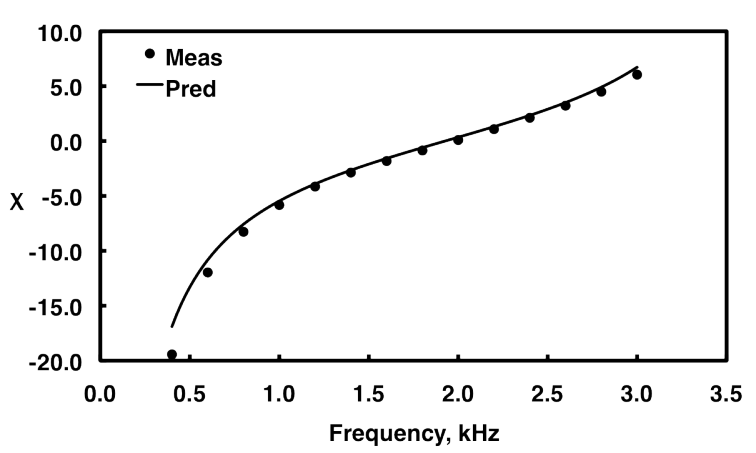

(b) Normalized reactance, $\chi$.

Figure 11: Predicted and measured normalized impedance spectra; narrow-chamber liner NC1.

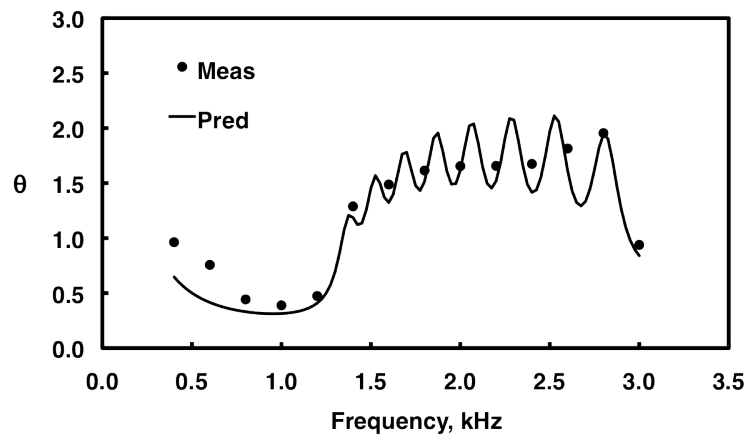

(a) Normalized resistance, $\theta$.

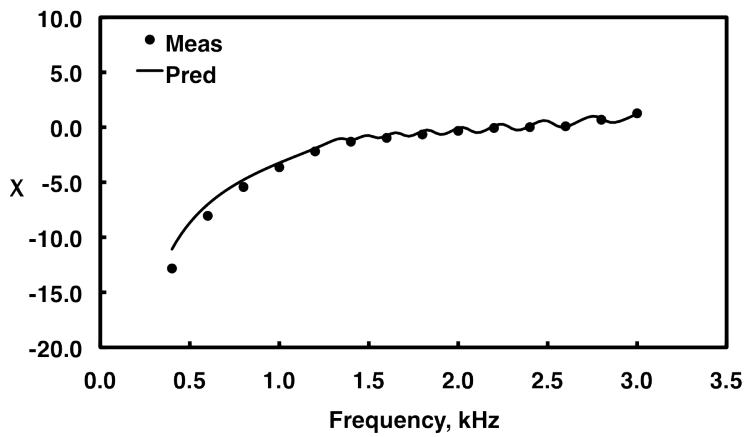

(b) Normalized reactance, $\chi$.

Figure 12: Predicted and measured normalized impedance spectra; narrow-chamber liner NC2.

The second narrow-chamber liner ( $\mathrm{NC} 2$ ) consists of a group of 9 narrow chambers with depths ranging from 1.12 to 2.41 inches. This group of chambers is repeated thirty times to form the complete 2 -in $\times 2$-in liner. The liner was designed to maximize absorption of normally incident sound over the frequency range of 1.4 to $3.0 \mathrm{kHz}$. Figure 12 provides a comparison of the predicted and measured impedance spectra for this liner. The variable chamber depths cause the resistance spectrum to deviate significantly from that observed for the constant-depth liner. The comparison between the predicted and measured resistance spectra is very good, and the corresponding comparison for reactance is even better. An interesting feature is observed in the resistance spectrum of Fig. 12. The choice of nine unique chamber depths results in nine peaks in the full resistance spectrum, as there is a distinct resonance frequency associated with each chamber depth. Only eight of these peaks are observed in Fig. 12a, as the highest resonance frequency is at a frequency outside the range shown. It should be noted that the measured data in Fig. 12 could easily be "fit" with lower-order polynomials. However, separate tests with a number of variable-depth liners provide added confidence in the predictions, i.e., demonstrate the validity of the oscillatory nature of the resistance and reactance spectra.

Figure 13 provides a comparison of measured absorption coefficient spectra for these narrow-chamber liners. The symbols represent the measured data, and the lines have been added as a graphical aid. As expected, the constant-depth liner ( $\mathrm{NC1}$ ) is a narrow-band absorber designed for $2 \mathrm{kHz}$. The variable-depth liner (NC2) is a broadband absorber, with an absorption coefficient of at least 0.7 over the design frequency range of 1.4 to $3.0 \mathrm{kHz}$, which was the target frequency range for this design. This result was expected, as it has previously been demonstrated that the impedance of this type of liner can be accurately predicted. ${ }^{6}$ Therefore, the quality of these comparisons can be taken as confirmation of the process used to fabricate these liners. Recall that the earlier tests were conducted with ceramic tubular material, which is quite heavy and brittle. The current liners are fabricated with plastic material and, although still unsuitable for use in 


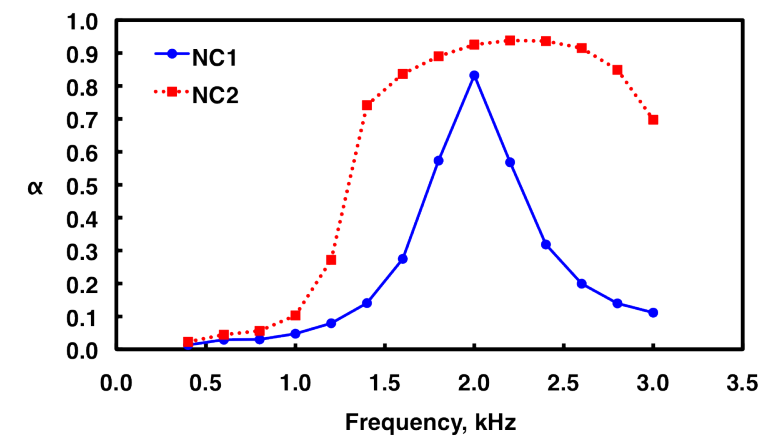

Figure 13: Comparison of measured absorption coefficient spectra; narrow-chamber liners NC1 and NC2.

aircraft engine nacelles, are much more robust for laboratory usage (e.g., flow ducts and model scale engine tests). Given the fidelity of the impedance prediction model, this type of liner is well suited for tailoring of the impedance spectrum.

\section{B. Parallel-element, variable-depth, wide-chamber liners}

As described in Tables 2 and 3, there are a number of wide-chamber liner configurations that were considered. The liner cores and facesheets are independent structures, thereby allowing convenient interchange of components for the evaluation of multiple liner configurations. Results are provided for two cores, one with constant-depth chambers (WC1) and the other with variable-depth chambers (WC2), each combined with facesheets F1 through F4.

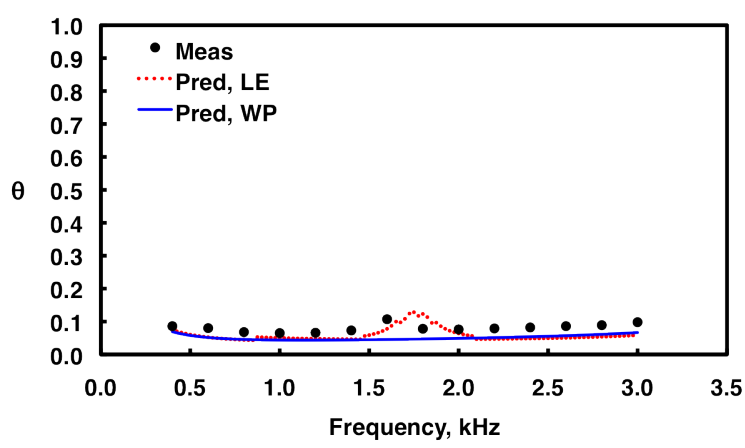

(a) Normalized resistance, $\theta$.

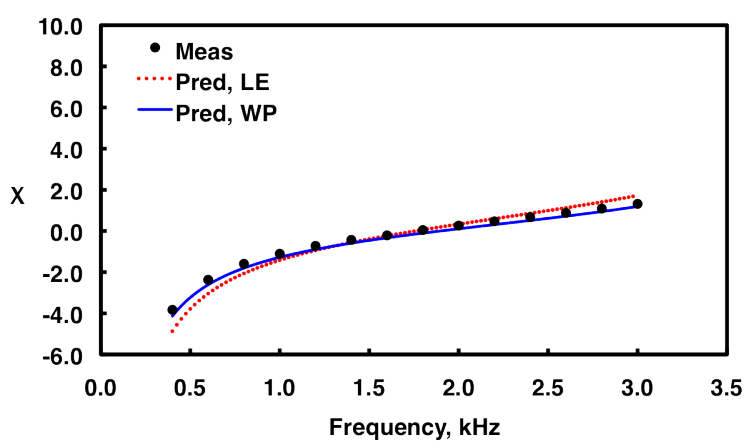

(b) Normalized reactance, $\chi$.

Figure 14: Predicted and measured normalized impedance spectra; wide-chamber core WC1, facesheet F1. Predicted spectra provided for lumped element (LE) and wave propagation (WP) models.

Figure 14 provides a comparison of measured and predicted impedance spectra for the WC1F1 liner (WC1 core with F1 facesheet). The scales of the resistance, $\theta$, and reactance, $\chi$, plots are chosen to allow comparison with successive figures. As the chamber are quite large $(0.375$-in $\times 0.375$-in square chambers), scrubbing losses along the interior walls of the chambers provide minimal resistance. These square chambers are represented with their equivalent diameter $\left(d_{c}\right.$ is given by the diameter of a circular chamber with the same cross-sectional area) in the impedance prediction model. Facesheet F1, with a thickness of 0.025 -in, provides a measured resistance of approximately 0.1 across the entire frequency range of interest. As this liner contains a perforated sheet, both modeling approaches (lumped element and wave propagation, labeled as "LE" and "WP", respectively) are considered. Minimal differences are observed between the resistance spectra predicted with each approach, but the lumped element approach captures the slight increase in measured resistance near resonance. The measured reactance spectra follows the expected $-\cot (k h)$ pattern. Also, 
although both modeling approaches provide very similar predicted reactance spectra, the wave propagation approach provides a slightly better match to the measured reactance spectrum, especially at the lower frequencies.

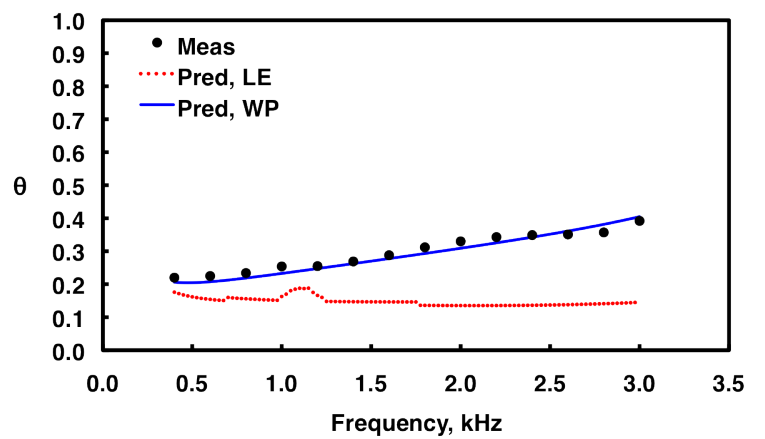

(a) Normalized resistance, $\theta$.

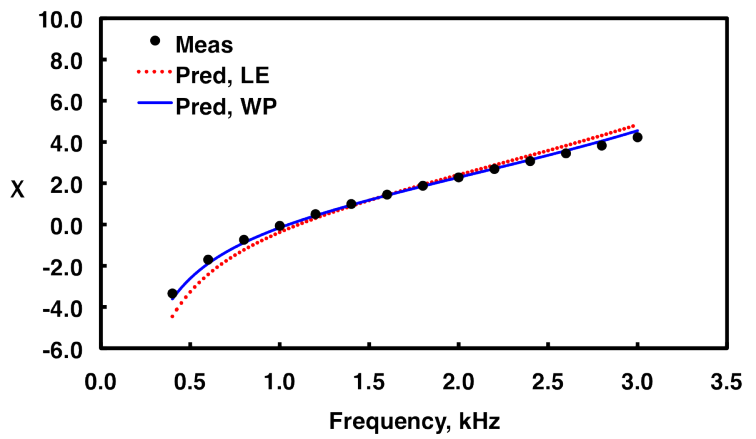

(b) Normalized reactance, $\chi$.

Figure 15: Predicted and measured normalized impedance spectra; wide-chamber core WC1, facesheet F4.

Figure 15 provides the corresponding measured and predicted impedance spectra for the WC1F4 liner (WC1 core with F4 facesheet). The only difference between this liner and the WC1F1 liner is the thickness of the facesheet. The F4 facesheet has a thickness of 0.200-in, which is significantly larger than those used for conventional liners. The semi-empirical lumped element model was developed for conventional liners, so the difference between the LE prediction and the measured resistance spectrum seems reasonable. On the other hand, the increased thickness of the facesheet is expected to support wave propagation within the individual orifices of the facesheet. This premise is supported by the excellent comparison of the WP prediction and the measured resistance spectrum. As with the WC1F1 liner, both models compare favorably with the measured reactance spectrum, although the wave propagation model provides the best comparison.

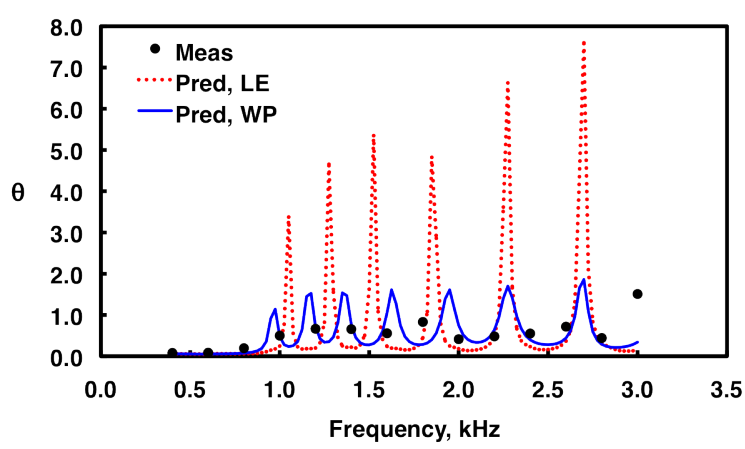

(a) Normalized resistance, $\theta$.

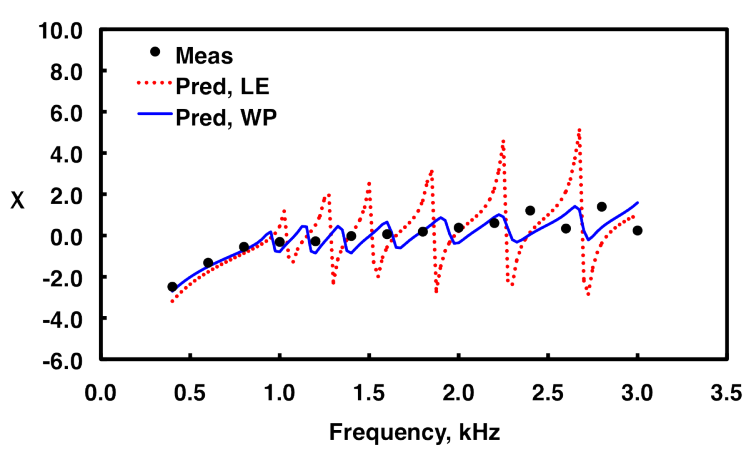

(b) Normalized reactance, $\chi$.

Figure 16: Predicted and measured normalized impedance spectra; wide-chamber core WC2, facesheet F1.

Figures 16 and 17 provide the results for the WC2F1 (WC2 core with $\mathrm{F} 1$ facesheet) and WC2F4 (WC2 core with F4 facesheet) liners. Recall that the WC2 core contains seven unique chamber depths (see Tab. 2). As with the variable-depth, narrow chamber sample, seven distinct chamber depths are expected to provide seven resonant frequencies. The figure shows six and seven peaks in the acoustic resistance spectra predicted with the LE and WP models, respectively. The seventh peak for the LE prediction occurs just outside the frequency range of interest. Although the two predicted resistance spectra differ significantly, both models compare favorably with the measured data at the measurement frequencies for the liner with the F1 (0.025-in thickness) facesheet (Fig. 16a). When the thickness of the facesheet is increased to 0.200 inches (F4 facesheet; see Fig. 17a), all but two of the peaks in the WP predicted resistance spectrum disappear, while the LE predicted resistance spectrum experiences minimal changes. The WP model comparison with the measured 
resistance spectrum is clearly much better than that achieved with the LE model. The corresponding reactance comparisons are fairly similar. Both prediction models compare favorably with the measured reactance spectrum for the thin facesheet, but the WP model provides much better comparison than the LE model for the thick facesheet.

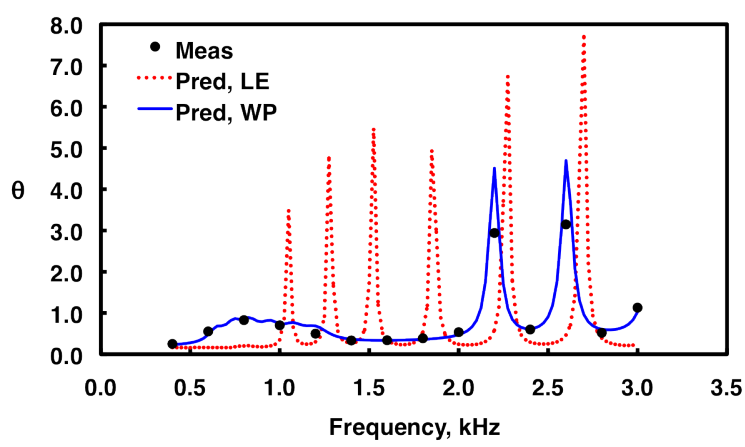

(a) Normalized resistance, $\theta$.

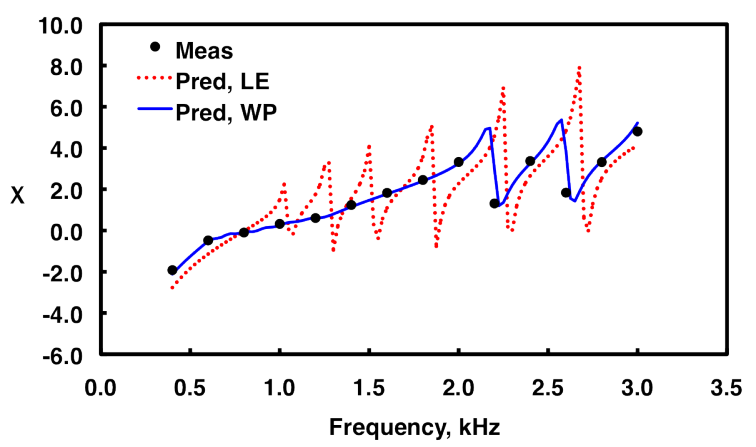

(b) Normalized reactance, $\chi$.

Figure 17: Predicted and measured normalized impedance spectra; wide-chamber core WC2, facesheet F4.

Figure 18 provides a comparison of absorption coefficient spectra measured with each of the four facesheets (F1 - F4; see Tab. 3) mounted onto the variable-depth (WC2) core. The symbols represent the measured data, and the lines have been added as a graphical aid. Each of these liner configurations provides excellent sound absorption over an extended frequency range. As the facesheet thickness is increased, the peak of the absorption coefficient spectrum shifts to lower frequencies, and the frequency region of high absorption decreases.

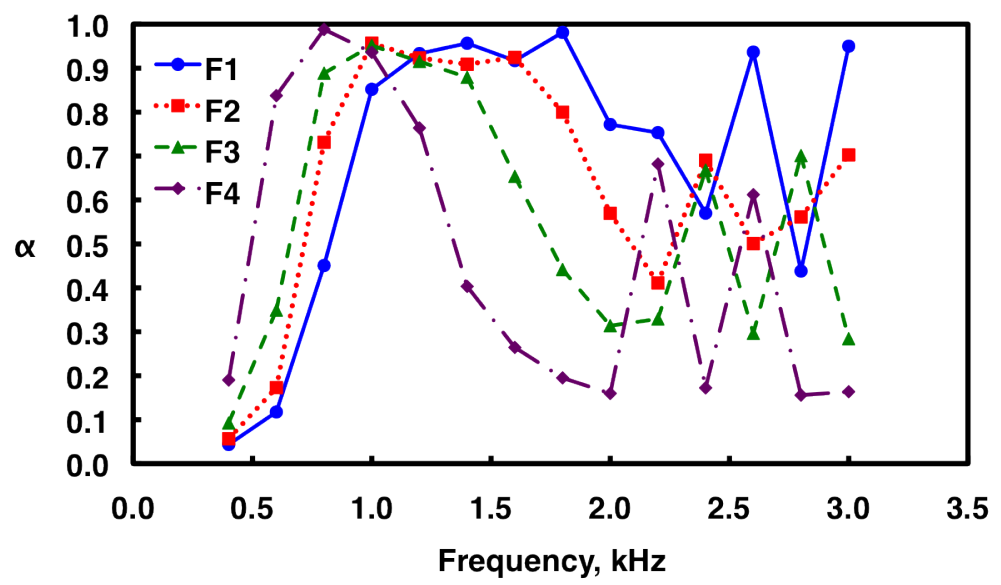

Figure 18: Facesheet thickness effects on measured absorption coefficient spectra; wide-chamber core WC2.

\section{Multi-layer liner with mesh-caps}

Results are presented for two mesh-cap liners (MC1 and MC2; see Tab. 4). As mentioned earlier, these liners do not contain facesheets (brass plate used for backplate). The first liner (MC1) contains one embedded mesh-cap, and the second (MC2) contains two embedded mesh-caps. These mesh-caps are very thin, and are thus expected to be amenable to prediction via the lumped element model. Figures 19 and 20 provide comparisons of measured and predicted impedance spectra. The measured resistance spectra increase with frequency for both configurations. The measured reactance spectra follow a $-\cot (k h)$ pattern for the lower portion of the frequency range, and then flatten at the higher frequencies. 


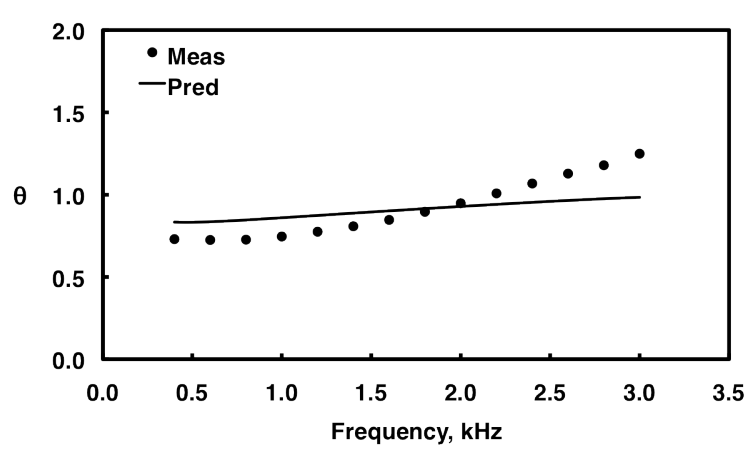

(a) Normalized resistance, $\theta$.

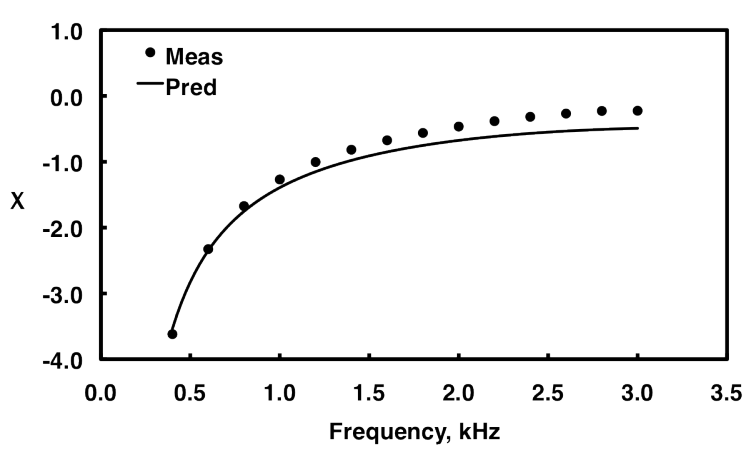

(b) Normalized reactance, $\chi$.

Figure 19: Predicted and measured normalized impedance spectra; mesh-cap liner MC1.

The mesh caps do not have a constant insertion depth (see Fig. 6) across the width of a single honeycomb chamber (the mesh has a "U-shape"). As used in this study, the lumped element model does not account for this insertion depth variability within an individual chamber. Instead, the insertion depth at the center of the chamber is used as the septum location for the predictions. There is therefore some uncertainty in the DC flow resistance of the mesh material. Given these factors, the comparisons of measured and predicted impedances are quite good (note the expanded scales).

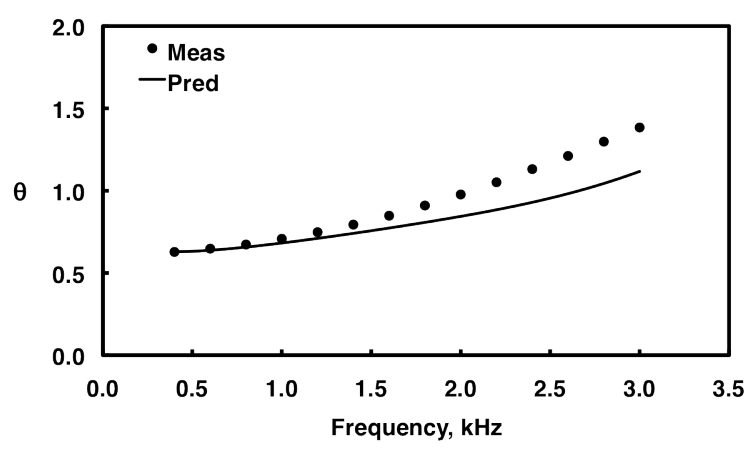

(a) Normalized resistance, $\theta$.

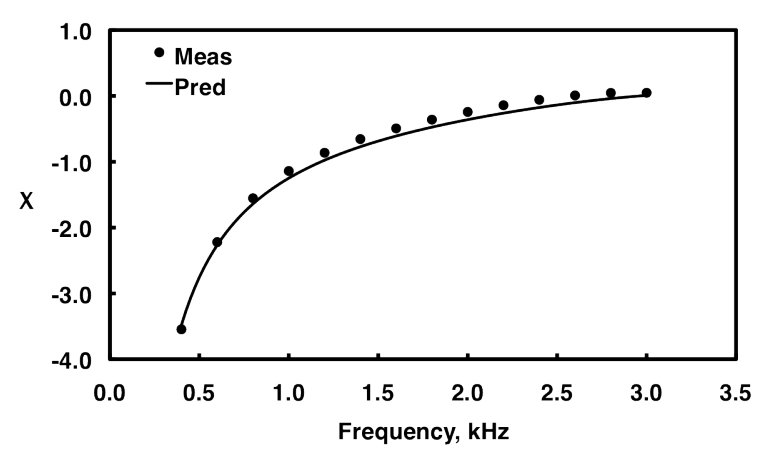

(b) Normalized reactance, $\chi$.

Figure 20: Predicted and measured normalized impedance spectra; mesh-cap liner MC2.

Figure 21 provides a comparison of absorption coefficient spectra measured for each of the mesh-cap liners. Both are observed to provide excellent sound absorption over the design frequency range (1.4 to $3.0 \mathrm{kHz}$ ). Recall that these liners did not contain facesheets. Thus, it is reasonable to assume that the addition of a facesheet should cause similar effects to those observed for the wide-chamber liners (i.e., shift frequency range of peak absorption downward and reduce absorption bandwith).

\section{Concluding Remarks}

Recent trends in aircraft engine design have caused the emphasis of fan noise control to transition from tonal to broadband. Aircraft manufacturers have addressed this issue by the use of liners with multiple layers (i.e., a perforate-over-honeycomb liner with an embedded porous septum), which provide a broadening of the frequency range for peak sound absorption. The width of the sound absorption frequency range increases with each additional layer, but limits on the liner thickness currently restrict conventional liners to one or two layers. It is therefore imperative that alternative acoustic liner concepts are developed to increase the sound absorption frequency range while maintaining or reducing the liner thickness.

This study has presented three liner concepts that offer this potential. Each of these liner concepts was 


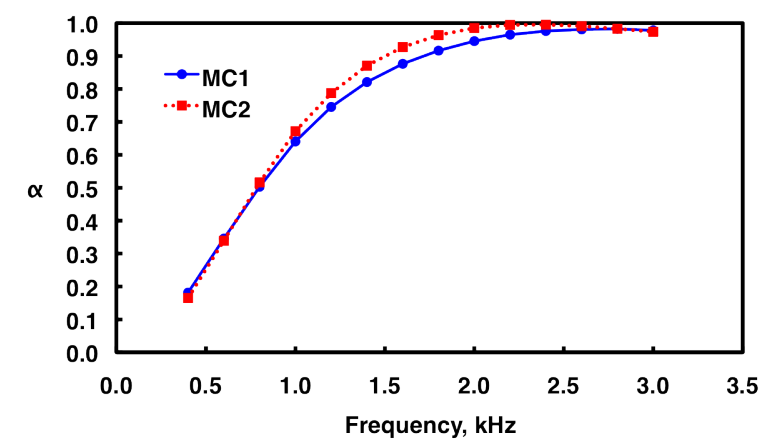

Figure 21: Comparison of measured absorption coefficient spectra; mesh-cap liners MC1 and MC2.

evaluated via tests using the NASA normal incidence impedance tube. Hence, the results provided herein do not include the effects of grazing flow. Those liner concepts that provide desirable sound absorption characteristics in the no-flow environment will be further evaluated in the NASA flow ducts, and the results will be provided in future reports.

Two of the liner concepts employ additive manufacturing techniques to fabricate liners with variable chamber depths. The first relies on narrow chambers to provide the acoustic resistance necessary for sound absorption, and achieves a near-zero reactance across a broad frequency range via the combination of chambers with variable depths. The second also uses variable-depth chambers to achieve a near-zero reactance spectrum, but the resistance is provided via a perforated facesheet. These wide-chamber liners are lighter than their narrow-chamber counterparts, and therefore appear more suitable for aircraft applications. As advances in material development continue, each of these concepts should be considered further for potential usage in aircraft applications. In the meantime, both can be used to achieve targeted impedance spectra for usage in laboratory and model scale engine tests.

The third liner concept employs embedded mesh-caps (resistive sheets) to create variable-impedance liners by varying the insertion depths of the mesh-caps between chambers. Liners fabricated with mesh-caps inserted at a constant depth are currently employed in aircraft engine nacelles. Thus, it appears obvious that the variable-impedance liner configurations considered in this study are amenable to aircraft applications.

Each of these liner concepts can be used to provide broadband sound absorption. Perhaps more importantly, the transmission line impedance prediction model presented herein provides good to excellent comparisons with measured impedance spectra for each liner concept. For those liners with facesheets, the current study has demonstrated that a modeling approach that employs a lumped element facesheet model is acceptable for the thin facesheets currently in use. Another modeling approach that allows for wave propagation within the perforates of the facesheet also compares favorably with measured results for liners with thin facesheets. As the facesheet thickness is increased, the lumped element model fails, but the wave propagation model continues to provide favorable comparisons with measured results.

It is also of interest to note that the use of a facesheet with increased thickness causes the frequency of peak sound absorption to decrease, and reduces the bandwidth of high sound absorption. Recall that one of the research goals is to develop liner concepts that achieve broadband sound absorption with reduced liner thickness. The current results achieved with a thick facesheet suggest the possibility of one such solution. If the chamber lengths of a wide-chamber, variable-depth liner are all decreased, the frequency range of optimum sound absorption will shift toward higher frequencies. If a properly designed thick facesheet is employed with this liner, it is postulated that this shift of the optimum frequency range could be offset. Further evaluation of this concept, including the effects of grazing flow, is expected to be the subject of a future study.

\section{Acknowledgements}

The authors would like to express appreciation to Carol Harrison of NASA Langley Research Center, Clark Smith and Fumitaka Ichihashi of Hexcel Corporation, and Jim Buckley of ASRC Aerospace Corpo- 
ration for their contributions to this study. This work was funded by the Subsonic Fixed Wing Project of NASA's Fundamental Aeronautics Program and the Environmentally Responsible Aviation Project of NASA's Integrated Systems Research Program. A portion of this study was conducted as part of a Space Act Agreement between NASA and Hexcel Corporation.

\section{References}

${ }^{1}$ Lockard, D. P. and Lilley, G. M., "The Airframe Noise Reduction Challenge," NASA TM 213013, 2004.

${ }^{2}$ Envia, E. E., Huff, D. L., and Morrison, C. R., "Analytical assessment of stator sweep and lean in reducing rotor-stator tone noise," AIAA Paper 1996-1791, May 1996.

${ }^{3}$ Woodward, R. P., Elliott, D. M., Hughes, C. E., and Berton, J. J., "Benefits of Swept-and-Leaned Stators for Fan Noise Reduction," Journal of Aircraft, Vol. 38, No. 6, 2001, pp. 1130-1138.

${ }^{4}$ Bielak, G. W., Premo, J. W., and Hersh, A. S., "Advanced Turbofan Duct Liner Concepts," NASA CR $209002,1999$.

${ }^{5}$ Wirt, L. S., "Analysis, Testing, and Design of Lined Ducts," Journal of the Acoustical Society of America, Vol. 51, No. 5, 1972, pp. 1448-1463.

${ }^{6}$ Parrott, T. L. and Jones, M. G., "Parallel-Element Liner Impedances for Improved Absorption of Broadband Sound in Ducts," Noise Control Engineering Journal, Vol. 43, No. 6, November - December, 1995.

${ }^{7}$ Syed, A. A. and Ichihashi, F., "The Modeling and Experimental Validation of the Acoustic Impedance of Multi-Degreesof-Freedom Liners," AIAA Paper 2008-2927, May 2008.

${ }^{8}$ Chung, J. Y. and Blaser, D. A., "Transfer function method of measuring in-duct acoustic properties: I. Theory," Journal of Acoustical Society of America, Vol. 68, 1980, pp. 907-921.

${ }^{9}$ Jones, M. G. and Parrott, T. L., "Evaluation of a multi-point method for determining acoustic impedance," Journal of Mechanical Systems and Signal Processing, Vol. 3, No. 1, 1989, pp. 15-35.

${ }^{10}$ Jones, M. G., Watson, W. R., and Parrott, T. L., "Benchmark Data for Evaluation of Aeroacoustic Propagation Codes with Grazing Flow," AIAA Paper 2005-2853, May 2005.

${ }^{11}$ Jones, M. G., Parrott, T. L., and Watson, W. R., "Uncertainty and Sensitivity Analyses of a Two-Parameter Impedance Prediction Model," AIAA Paper 2008-2928, May 2008. 